\title{
Involvement of CXC Chemokine Growth-Related Oncogene- $\alpha$ in Monosodium Urate Crystal-Induced Arthritis in Rabbits
}

\author{
Kazunori Fujiwara, Susumu Ohkawara, Katsumasa Takagi, Masaru Yoshinaga, and \\ Akihiro Matsukawa
}

Departments of Pathology (KF, SO, MY, AM) and Orthopedics (KF, KT), Kumamoto University School of Medicine, Kumamoto, Japan

SUMMARY: Accumulation of neutrophils is a prominent feature of gouty arthritis in which CXC chemokines may play a role. Recently, we have shown that IL-8 (CXCL8) contributes to neutrophil influx in a rabbit model of gouty arthritis. Here, we demonstrate that growth-related oncogene- $\alpha(\mathrm{GRO} \alpha)(\mathrm{CXCL} 1)$, a prototype of CXC chemokine, is also involved in this process. $\mathrm{GRO} \alpha$ level in the joints peaked at 2 hours after intra-articular injection of monosodium urate crystals, at a time before the neutrophil influx reached the maximal level (9 hours). Once decreased, the level increased and reached the second peak at 9 hours. The kinetics was comparable to that of IL-8. Administration of anti-GRO $\alpha \mathrm{mAb}$ attenuated the neutrophil influx at the same level as did the anti-IL-8 IgG, and combination of these antibodies enhanced the inhibition, resulting in a $33 \%$ reduction. Interaction of $\mathrm{GRO} \alpha$ with $\mathrm{TNF} \alpha, \mathrm{IL}-1 \beta$, and IL-8 was next investigated by injecting antibodies or receptor antagonist with monosodium urate crystals. Administration of anti-TNF $\alpha$ mAb did not alter GRO $\alpha$ level at 2 hours, but inhibited the levels 9 hours after the injection. Treatment with either IL-1 receptor antagonist or anti-IL-8 IgG resulted in decreased levels of GRO $\alpha$ at 2 and 9 hours. Neutralization of GRO $\alpha$ with anti-GRO $\alpha$ mAb did not alter TNF $\alpha$, IL-1 $\beta$, and IL- 8 levels at their peak ( 2 hours), but decreased the second peak of IL-1 $\beta$ (9 hours) and IL-8 (12 hours). These results provide evidence that GRO $\alpha$ as well as IL-8 are involved ad eundem in the neutrophil infiltration in this model. IL-1 and IL-8, but not TNF $\alpha$, are responsible in part for the initial phase of $\mathrm{GRO} \alpha$, whereas these cytokines induce $\mathrm{GRO} \alpha$ in a late phase. GRO $\alpha$ does not seem to initiate TNF $\alpha$, IL-1 $\beta$, and IL- 8 in an early phase, but induces IL-1 $\beta$ and IL-8 in a late phase. (Lab Invest 2002, 82:1297-1304).

G out is characterized by recurrent episodes of acute arthritis with redness, swelling, and intense pain of the joint, in which shedding of monosodium urate (MSU) crystals from preexisting articular deposits into joint cavities triggers an acute inflammation (Alexander, 1986). Studies have shown that an initial interaction between MSU crystals and cells in the joints is essential for the initiation and development of acute gouty arthritis. MSU crystals activate synovial cells, which release inflammatory mediators, leading to the accumulation of cells, neutrophils in particular, from the circulation. Once activated, the cells further release various inflammatory mediators that intensify the inflammatory responses (Gordon et al, 1988; McCarty, 1994). A wide variety of inflammatory mediators can be produced in the joints, including histamine, leukotriene B4, prostaglandins, platelet ac-

DOI: 10.1097/01.LAB.0000029206.27080.D2

Received April 9, 2002.

This work was supported in part by grants from the Ministry of Education, Science, Sports and Culture, and The Ministry of Health and Welfare, Japan.

Address reprint requests to: Dr. A. Matsukawa, Department of Pathology, Kumamoto University School of Medicine, 2-2-1, Honjo, Kumamoto 860-0811, Japan. E-mail: matsu@kaiju.medic.kumamoto-u.ac.jp tivating factor, proinflammatory cytokines, and chemokines (Terkeltaub, 1993; Weinberger, 1995).

Among chemokines, evidence suggests that the CXC chemokines play critical roles in the accumulation of neutrophils (Baggiolini et al, 1997; Rollins, 1997). The CXC chemokines are a structurally related and functionally redundant family of proteins with specific leukocyte chemotactic activity that can be divided into two subsets based on the presence or absence of specific amino acid residues Glu-Leu-Arg (ELR) (Baggiolini et al, 1997; Zlotnik and Yoshie, 2000). IL-8 (CXCL8) and growth-related oncogene- $\alpha$ (GRO $\alpha$ : CXCL1) are members of ELR CXC chemokines that attract neutrophils. These chemokines have been detected in a variety of clinical diseases characterized by a massive neutrophil infiltration that include acute respiratory distress syndrome, bacterial inflammation, and rheumatoid arthritis (Kunkel et al, 1995; Luster, 1998). We have shown that these chemokines are essential in the recruitment of neutrophils in animal models of inflammation. Equivalent levels of IL-8 and $\mathrm{GRO} \alpha$ were detected at the foci of LPS-inflammation, and blockade of each chemokine resulted in a decrease in the number of infiltrating neutrophils. The inhibition was enhanced when these chemokines were together neutralized (Fukumoto et al, 1998; Matsukawa et al, 1997, 1998; Mo et al, 1999, 2000). 
A large amount of IL-8 was detected in the synovial fluids of patients with gout (Terkeltaub et al, 1991). We and others have shown that IL-8 is involved in the neutrophil infiltration in a rabbit model of gouty arthritis (Matsukawa et al, 1998; Nishimura et al, 1997), suggesting an important role of IL-8 in gouty arthritis. However, little is known about the role of $\mathrm{GRO} \alpha$ in gouty arthritis. The aim of the present study was to elucidate the role of $\mathrm{GRO} \alpha$ and its possible cooperation with IL-8 in gouty arthritis. To address this, rabbits received intra-articular injection of MSU crystals and the subsequent inflammatory responses were investigated. We also analyzed the interaction of $\mathrm{GRO} \alpha$ with TNF $\alpha$, IL-1 $1 \beta$, and IL-8 in this model of gouty arthritis.

\section{Results}

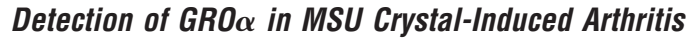

Intra-articular injection of MSU crystals resulted in an increase in the synovial level of $\mathrm{GRO} \alpha$. $\mathrm{GRO} \alpha$ was first detectable at 1 hour and peaked at 2 hours after the injection. Once decreased at 4 hours, the level increased again and reached a second peak at 9 hours, after which the level decreased gradually and returned to the basal level at 36 hours (Fig. 1). We previously demonstrated the production of IL-8 in this model (Matsukawa et al, 1998). To compare the kinetics between these chemokines, the IL-8 level was measured in these newly harvested samples. As shown in Figure 1, the kinetics of IL-8 was similar to that of $\mathrm{GRO} \alpha$, peaking at 2 and 12 hours after the injection. Interestingly, GRO $\alpha$ level was higher than that of IL-8, demonstrating a two-fold increase at 2 hours (Fig. 1).

The cell-associated GRO $\alpha$ level was also measured quantitatively. The data showed that infiltrating leukocytes contained abundant GRO $\alpha$ (Table 1). The peak level of $\mathrm{GRO} \alpha$ in the infiltrating leukocytes was ob-

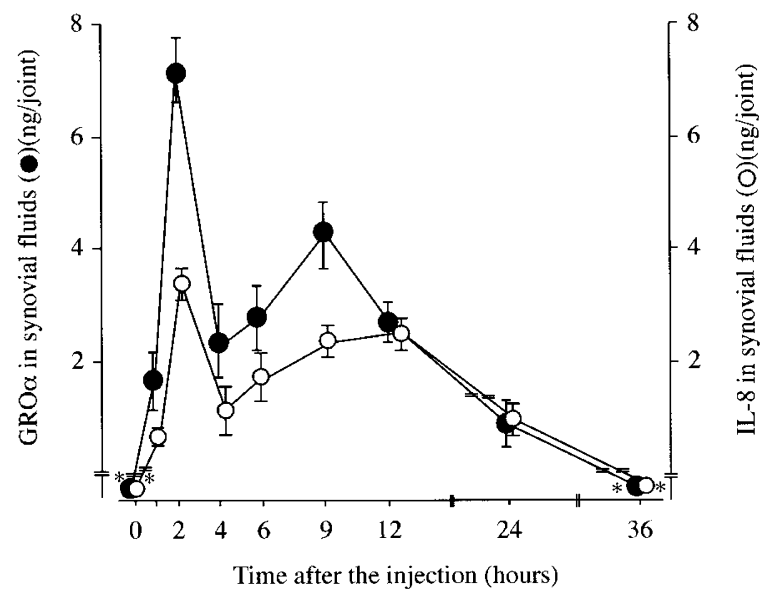

Figure 1.

Generation kinetics of GRO $\alpha$ and IL-8 in MSU-induced arthritis. At indicated times after the intra-articular injection of $10 \mathrm{mg}$ of monosodium urate (MSU) crystals, rabbits were killed. The joints were washed with saline and cleared synovial fluids were harvested. The levels of GRO $\alpha$ (closed circles) and IL-8 (open circles) in the samples were then measured. Each point represents the mean \pm SE of 8 to 30 estimations from separate joints. * indicates below detection level.
Table 1. Production of GRO $\alpha$ in Synovial Exudate Cells of MSU Crystal-Induced Arthritis

\begin{tabular}{cc}
\hline Time (hours) & GRO $\alpha$ (ng/joint) \\
\hline 2 & $0.7 \pm 0.1$ \\
4 & $4.0 \pm 0.5$ \\
6 & $9.9 \pm 0.9$ \\
9 & $13.2 \pm 1.0$ \\
12 & $8.8 \pm 1.2$ \\
24 & $2.1 \pm 0.3$ \\
\hline
\end{tabular}

At indicated times after intra-articular injection of $10 \mathrm{mg}$ of MSU crystals, the infiltrating leukocytes in the joints were collected and extracted. The levels of GRO $\alpha$ in the extracts were measured by time-resolved fluoroimmunoassay. Results are the mean \pm SE of findings in 8 to 12 separate joints.

served at 9 hours after MSU crystal injection, at a time when the number of infiltrating leukocytes (neutrophils $>95 \%$ ) reached the maximal level (Matsukawa et al, 1998). The $\mathrm{GRO} \alpha$ level in the infiltrating leukocytes was notably lower than that in the synovial fluids at 2 hours, but significantly higher after 4 hours (Fig. 1; Table 1). In neutrophil-depleted rabbits, the synovial level of $\mathrm{GRO} \alpha$ at 2 hours was comparable to that in control rabbits, whereas the $\mathrm{GRO} \alpha$ level at 9 hours was significantly lower than control (Fig. 2), suggesting that synovial resident cells were the main source of

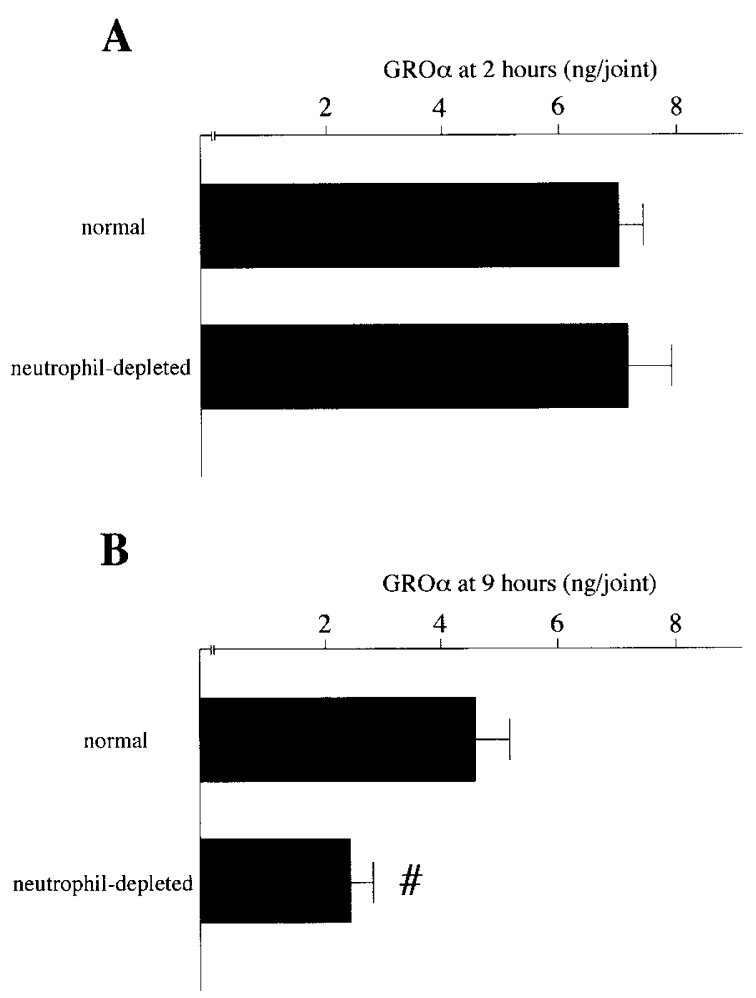

Figure 2.

GRO $\alpha$ level in normal and neutrophil-depleted rabbits. Neutrophil-depleted rabbits were prepared by intravenous injection of vinblastine $(0.75 \mathrm{mg} / \mathrm{kg})$. At 2 hours (A) and 9 hours (B) after the intra-articular injection of $10 \mathrm{mg}$ of MSU crystals, the joints were washed with saline, cleared synovial fluids were harvested, and the levels of GRO $\alpha$ in samples were measured. The data represent the mean \pm SE of 8 to 20 estimations from separate joints. \# $p<$ 0.05 when compared with normal rabbits. 
$\mathrm{GRO} \alpha$ in an early phase, whereas synovial resident cells and infiltrating leukocytes were responsible for $\mathrm{GRO} \alpha$ production in a late phase. Immunohistochemistry demonstrated that synovial lining cells were positively stained for GRO $\alpha$ at 2 hours (Fig. 3). These data clearly demonstrated that $\mathrm{GRO} \alpha$ was produced in the joints after MSU crystal injection.

\section{Involvement of GRO $\alpha$ and IL-8 in the Recruitment of Neutrophils}

To investigate the involvement of $\mathrm{GRO} \alpha$ and its cooperation with IL-8 in the recruitment of neutrophils in this model, anti-GRO $\alpha \mathrm{mAb}$, anti-IL-8 IgG, or their combination was administered together with MSU crystals, and the subsequent neutrophil infiltration was investigated. Administration of anti-GRO $\alpha$ mAb resulted in a decrease in the number of infiltrating neutrophils after 6 hours. A maximal level of neutrophil infiltration observed at 9 hours after the injection was inhibited by $16 \%$ (Fig. 4). Consistent with our previous observations (Matsukawa et al, 1998), administration of anti-IL-8 IgG attenuated the neutrophil infiltration, which was a $21 \%$ inhibition at 9 hours. When these antibodies were together injected with MSU crystals, the neutrophil infiltration was further inhibited, resulting in a 33\% reduction at 9 hours (Fig. 4). Thus, the contribution of $\mathrm{GRO} \alpha$ to the recruitment of neutrophils was comparable to IL-8, and GRO $\alpha$ attracted neutrophils in collaboration with IL-8.

\section{Regulation of GRO $\alpha$ by TNF $\alpha$, LL-1, and IL-8}

We previously demonstrated the production of $\operatorname{TNF} \alpha$, $\mathrm{IL}-1 \beta$, and IL-8 in this model, all of which peaked at 2 hours after MSU crystal injection (Matsukawa et al, 1998). To investigate whether these cytokines/chemokines could induce $\mathrm{GRO} \alpha$ in this model, anti-TNF $\alpha$ $\mathrm{mAb}, \mathrm{IL}-1$ receptor antagonist (IL-1Ra), or anti-IL-8 IgG was injected with MSU crystals. As shown in Figure 5, the GRO $\alpha$ level at 2 hours was not altered by anti-TNF $\alpha$ mAb, whereas the level was significantly inhibited (by $71 \%$ ) by IL-1Ra. Administration of antiIL-8 IgG also reduced the level, which was a $40 \%$ inhibition at 2 hours. On the other hand, either inhibitor decreased the second $\mathrm{GRO} \alpha$ peak seen at 9 hours after the injection (36\% by anti-TNF $\alpha \mathrm{mAb}, 32 \%$ by IL-1Ra, and $43 \%$ by anti-IL-8 IgG) (Fig. 5). These data

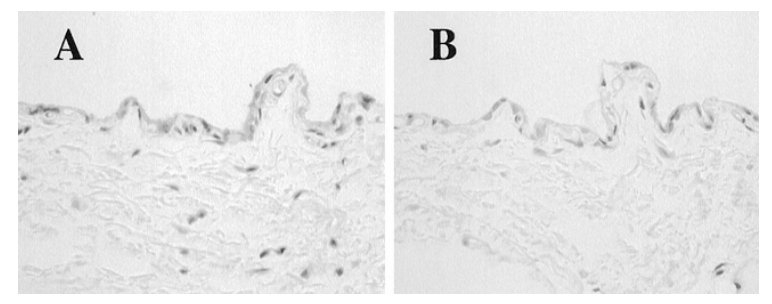

Figure 3.

Immunohistochemistry for GRO $\alpha$. Synovial tissues were obtained 2 hours after intra-articular injection of $10 \mathrm{mg}$ of MSU crystals. The sections were stained with anti-GRO $\alpha \operatorname{lgG}$, as described in the text. A, Synovial lining cells were positively stained for $\mathrm{GRO} \alpha$. B, No staining was found in control (preimmune IgG). Original magnification, $\times 1000$.

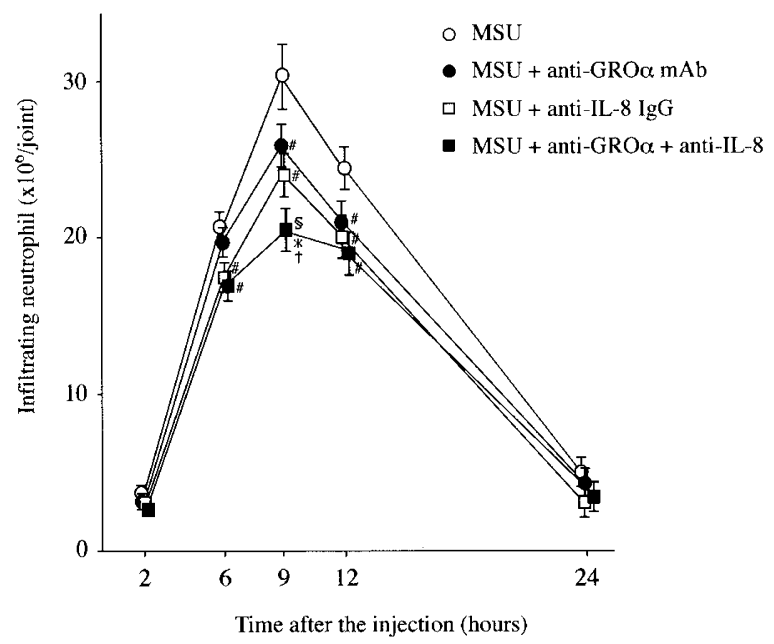

Figure 4.

Involvement of GRO $\alpha$ and IL-8 in neutrophil influx. Anti-GRO $\alpha \mathrm{mAb}$, anti-IL-8 IgG (10 $\mu \mathrm{g}$ each), or their combination was injected simultaneously with 10 $\mathrm{mg}$ of MSU crystals into rabbit knee joints. At indicated time intervals, the infiltrating leukocytes in the joints were counted. The data represent the mean \pm SE of 8 to 20 estimations from separate joints. $\# p<0.05, \S p<0.01$, when compared with MSU crystals alone. ${ }^{*} p<0.05$, when compared with MSU crystals + anti-GRO $\alpha$ mAb. $\dagger p<0.05$, when compared with MSU crystals + anti-IL-8 IgG.

demonstrated the differential regulation of $\mathrm{GRO} \alpha$ during inflammation.

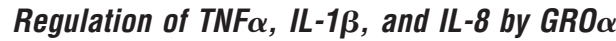

Experiments were next conducted to examine the role of $\mathrm{GRO} \alpha$ in the production of TNF $\alpha, \mathrm{IL}-1 \beta$, and IL-8. To do this, anti-GRO $\alpha$ mAb was injected with MSU crystals, and levels of TNF $\alpha$, IL- $1 \beta$, and IL- 8 were then measured at their peak (2 hours). Levels of IL-1 $\beta$ and IL-8 were also measured at their second peak ( 9 and 12 hours, respectively). The data demonstrated that administration of anti-GRO $\alpha \mathrm{mAb}$ failed to reduce the level of TNF $\alpha$, IL- $1 \beta$, and IL-8 at 2 hours after MSU crystal-injection (Fig. 6A). In contrast, the treatment significantly decreased the IL- $1 \beta$ level by $27 \%$ at 9 hours and the IL- 8 level by $23 \%$ at 12 hours (Fig. 6, B and C). Thus, production of TNF $\alpha, \mathrm{IL}-1 \beta$, and IL- 8 in an early phase was independent of $\mathrm{GRO} \alpha$, whereas $\mathrm{GRO} \alpha$ seemed to have a role in regulating the expression of both IL-1 $\beta$ and IL-8 in a late phase.

\section{Discussion}

In addition to IL-8, MSU crystals are capable of inducing $\mathrm{GRO} \alpha$ in vitro (Terkeltaub et al, 1991, 1998). Mice lacking CXC chemokine receptor 2 (CXCR2) had impaired neutrophil infiltration in the air pouch model of MSU crystal-induced inflammation (Terkeltaub et al, 1998). These results suggest that $\mathrm{GRO} \alpha$, a ligand for CXCR2, may be involved in the recruitment of neutrophils in gouty arthritis. In the present study, we demonstrated that $\mathrm{GRO} \alpha$ was detected in large concentrations in a rabbit model of gouty arthritis. Neutralization of $\mathrm{GRO} \alpha$ resulted in a decrease in the 


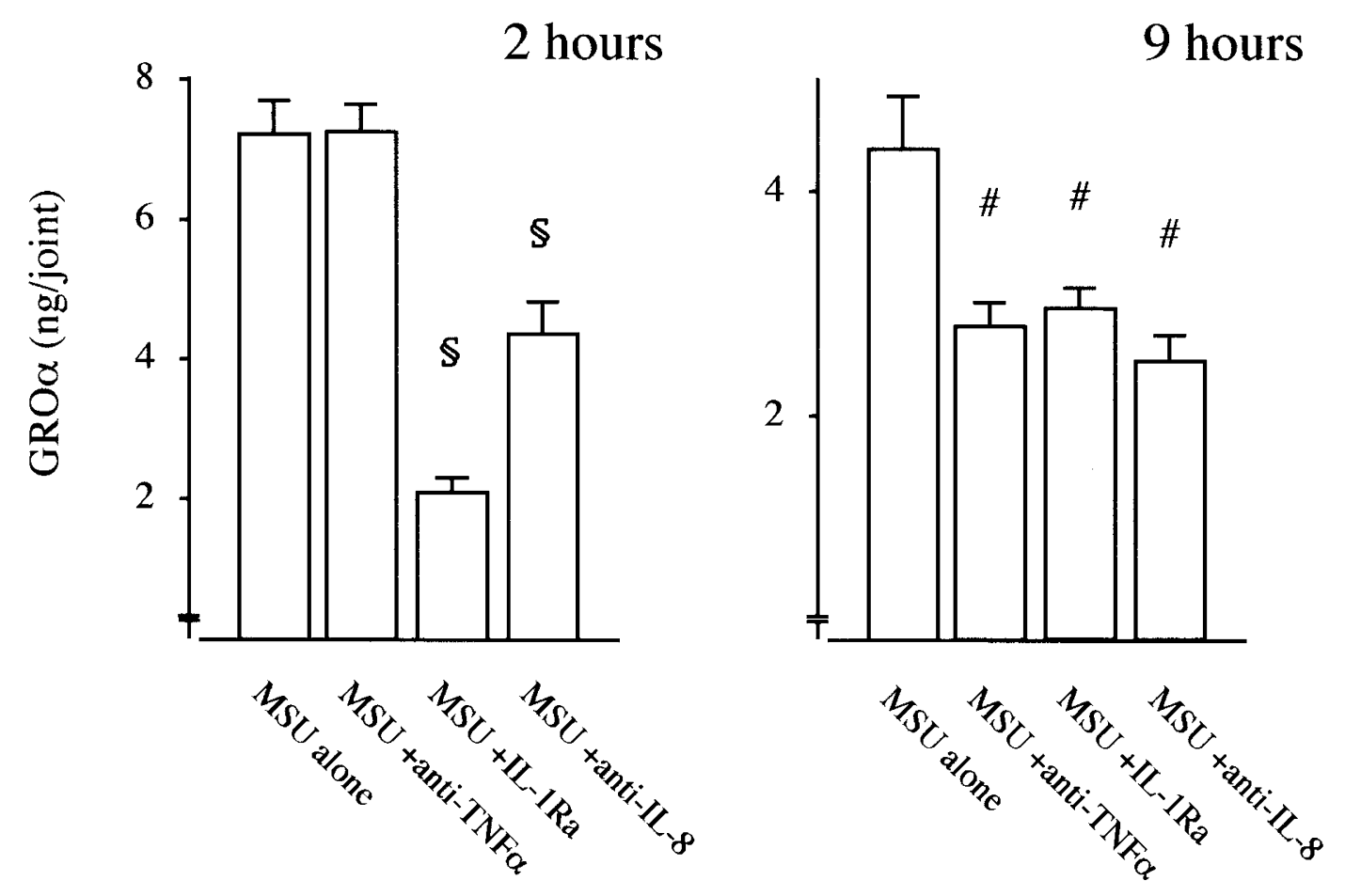

Figure 5.

Regulation of GRO $\alpha$ by TNF $\alpha$, IL-1, and IL-8. Anti-TNF $\alpha$ mAb, IL-1 receptor antagonist (IL-1Ra), or anti-IL-8 IgG (10 $\mu \mathrm{g}$ each) was injected with $10 \mathrm{mg}$ of MSU crystals into rabbit knee joints. At 2 hours and 9 hours after the injection, the synovial fluids were harvested and levels of GRO $\alpha$ were measured. The data represent the mean \pm SE of 8 to 20 estimations from separate joints. \# $p<0.05, \S p<0.01$, when compared with MSU crystals alone.

number of infiltrating neutrophils. The data clearly demonstrated the involvement of $\mathrm{GRO} \alpha$ in this model.

The participation of $\mathrm{GRO} \alpha$ in the recruitment of neutrophils was equivalent to IL-8, because the inhibition induced by anti-GRO $\alpha \mathrm{mAb}$ was comparable to that induced by anti-IL-8 IgG. Thus, GRO $\alpha$ seems to be involved in the recruitment of neutrophils at the same level as IL-8. However, the $\mathrm{GRO} \alpha$ level was two-fold higher than that of IL-8, suggesting that IL-8 might be more effective than $\mathrm{GRO} \alpha$ in attracting neutrophils. We recently demonstrated that the number of infiltrating neutrophils induced by IL-8 was more than that induced by $\mathrm{GRO} \alpha$, despite the same molar concentrations of chemokines injected into the joints (Fujiwara et al, 2002). This could be explained by the fact that IL-8 binds CXCR1 and CXCR2, whereas GRO $\alpha$ binds only CXCR2 (Hall et al, 1999).

When these antibodies were together injected with MSU crystals, the neutrophil infiltration was further inhibited, indicating that $\mathrm{GRO} \alpha$ and IL-8 acted in concert to mediate neutrophil infiltration. We had initially assumed that these two chemokines would be the main factors for neutrophil infiltration in this model. However, in contrast to our assumption, the participation of these chemokines in the recruitment of neutrophils in this model was relatively small; the maximal inhibition induced by these antibodies was only $33 \%$ at the peak. Likewise, neutrophil infiltration in an early phase was not altered with these antibodies. These results suggest that chemoattractants other than GRO $\alpha$ and IL-8 may play important role(s) in the accumulation of neutrophils. Webster et al (1972) demonstrated evidence for the combined actions of kinins, histamine, and complement in MSU crystalinduced inflammation model in the rat paw. Prostaglandins and leukotriene B4 were reported to play important roles in MSU crystal-induced arthritis (Rae et al, 1982; Rosenthale et al, 1972). A PAF antagonist exerted an anti-inflammatory effect in a rabbit model of gouty arthritis (Miguelez et al, 1996). Other mediators would be expected to include chemokines other than GRO $\alpha$ and IL-8 (Zlotnik and Yoshie, 2000). These mediators, probably acting in cooperation, are likely to be responsible for the other part of the neutrophil infiltration. Some of these seemed to be regulated by IL-8 and IL-1, because administration of anti-IL-8 IgG together with IL-1Ra resulted in a decrease in the number of neutrophils of up to $62 \%$ (Matsukawa et al, 1998). Further study is necessary to address these points.

We have analyzed the cytokine network among TNF $\alpha$, IL- $1 \beta$, and IL- 8 in LPS and MSU crystalinduced arthritis (Matsukawa et al, 1997, 1998). A characterization of $\mathrm{GRO} \alpha$ in the network was also investigated in LPS-induced arthritis and uveitis (Matsukawa et al, 1999; Mo et al, 2000). In MSU crystalinduced arthritis, production of $\mathrm{GRO} \alpha$ peaked at 2 hours, at the same time that $\operatorname{TNF} \alpha, \mathrm{IL}-1 \beta$, and IL- 8 peaked (Matsukawa et al, 1998), suggesting a possible interaction of $\mathrm{GRO} \alpha$ with $\mathrm{TNF} \alpha$, IL-1 $\beta$, and IL-8. Our present data suggest that TNF $\alpha$ does not initiate the production of $\mathrm{GRO} \alpha$ despite the production by synovial cells in vitro (Golds et al, 1989). Either IL-1 or IL- 8 blockade decreased the level of GRO $\alpha$ at 2 hours. 
A
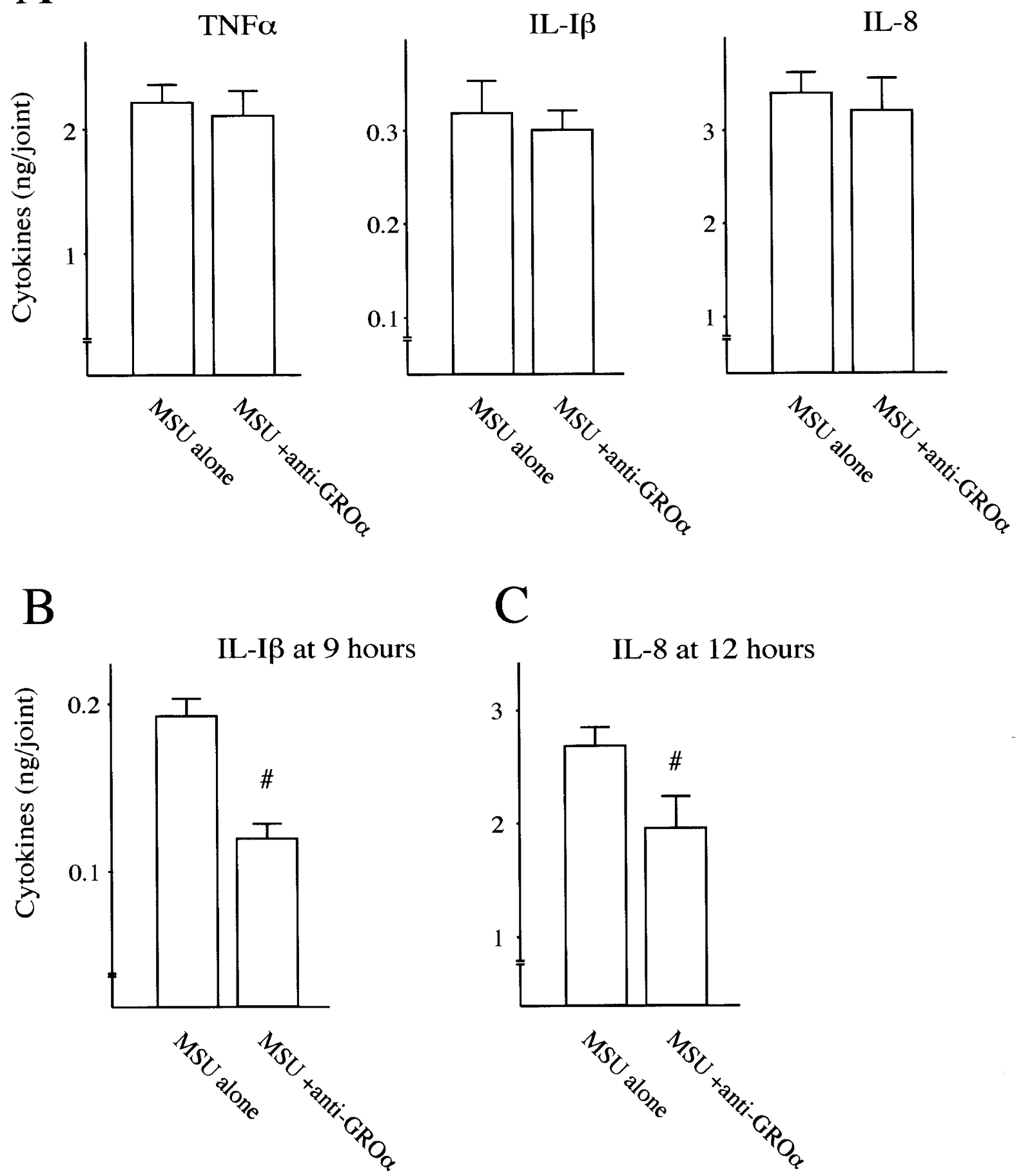

Figure 6.

Regulation of TNF $\alpha$, IL-1 $\beta$, and IL-8 by GRO $\alpha$. Anti-GRO $\alpha$ mAb (10 $\mu \mathrm{g})$ was injected with $10 \mathrm{mg}$ of MSU crystals into rabbit knee joints. At 2 hours (A), 9 hours $(B)$, and 12 hours (C) after the injection, the synovial fluids were harvested and levels of TNF $\alpha, I L-1 \beta$, and IL-8 were measured. The data represent the mean \pm SE of 8 to 20 estimations from separate joints. \# $p<0.05$, $§ p<0.01$, when compared with MSU crystals alone.

The effect of IL-8 seemed to be independent of IL-1, because anti-IL-8 IgG failed to reduce the level of $\mathrm{IL}-1 \beta$ at 2 hours (Matsukawa et al, 1998). Thus, GRO $\alpha$ seems to be regulated in part by IL-1 and IL-8, but not $\mathrm{TNF} \alpha$, in an initial phase. In a late phase, $\mathrm{GRO} \alpha$ level was reduced with anti-TNF $\alpha$ mAb, IL-1Ra, or anti-IL-8 IgG by $30 \%$ to $40 \%$. We demonstrated in our previous study that anti-TNF $\alpha$ mAb, IL-1Ra, or anti-IL-8 IgG decreased the infiltrating neutrophils (Matsukawa et al, 1998) at the same level as seen in $\mathrm{GRO} \alpha$ inhibition, and infiltrating neutrophils were another source of $\mathrm{GRO} \alpha$ in this phase. Thus, TNF $\alpha, \mathrm{IL}-1$, and IL- 8 seem to induce $\mathrm{GRO} \alpha$ in a late phase, indirectly via the accumulation of neutrophils. On the other hand, levels 
of TNF $\alpha, \mathrm{IL}-1 \beta$, and IL-8 at 2 hours were not altered with anti-GRO $\alpha \mathrm{mAb}$, indicating that these cytokines/ chemokine were independent of $\mathrm{GRO} \alpha$ in an initial phase. In contrast, anti-GRO $\alpha$ mAb decreased IL-1 $\beta$ and IL-8 level in a late phase. As shown in this study, administration of anti-GRO $\alpha$ mAb attenuated the neutrophil infiltration. Thus, the reduced IL- $1 \beta$ and IL-8 levels in a late phase by anti-GRO $\alpha \mathrm{mAb}$ were correlated with the decreased number of neutrophils at these time points by the treatment. We showed that infiltrating neutrophils were responsible for the production of IL- $1 \beta$ and IL-8 in a late phase (Matsukawa et al, 1998). Altogether, it is conceivable that $\mathrm{GRO} \alpha$ induces IL-1 $\beta$ and IL-8 production in a late phase indirectly via the accumulation of neutrophils. However, the degree of cytokine inhibition by anti-GRO $\alpha$ $\mathrm{mAb}(\mathrm{IL}-1 \beta, 27 \%$; IL-8, 30\%) was greater than that of neutrophil infiltration by the treatment $(16 \%$ at 9 hours and $15 \%$ at 12 hours). The discrepancy might be explained by the activation of neutrophils by $\mathrm{GRO} \alpha$; that is, GRO $\alpha$ may activate neutrophils, resulting in the stimulation of IL- $1 \beta$ and IL- 8 release from the cells.

Studies have built the concept that chemokines are crucial in the pathogenesis of inflammatory responses. In LPS-induced arthritis, IL-8 as well as $\mathrm{GRO} \alpha$ blockade resulted in a considerable decrease in the number of infiltrating neutrophils, which was a $65 \%$ and $70 \%$ inhibition at 2 and 9 hours, respectively (Matsukawa et al, 1997, 1999). In contrast, the participation of these chemokines to the neutrophil infiltration in MSU crystal-induced arthritis was limited, up to $33 \%$ at 9 hours. In addition, the neutrophil infiltration at 2 hours was unchanged by IL- 8 and GRO $\alpha$ blockade. Our series of experiments revealed the distinct contribution of these chemokines to the neutrophil infiltration in different models of inflammation.

\section{Materials and Methods}

\section{Reagents}

Anti-rabbit IL-8 and anti-rabbit GRO $\alpha$ IgG were raised in a goat, and purified with affinity column coupled with each chemokine (Matsukawa et al, 1997, 1999). Anti-rabbit $\mathrm{GRO} \alpha \mathrm{mAb}$ was prepared as described (Matsukawa et al, 1999). Anti-rabbit TNF $\alpha$ mAb was kindly provided by Dr. H. Nariuchi (University of Tokyo, Japan) (Haranaka et al, 1985). Monoclonal and polyclonal antibodies used in this study had specific neutralizing activity and did not cross-react with other rabbit cytokines/chemokines available (data not shown). Recombinant rabbit IL-1Ra was purified, as described previously (Matsukawa et al, 1992). Endotoxin contaminations in reagents were removed with Kurimover II (Kurita Kogyo Company, Tokyo, Japan), according to the manufacturer's instruction. Endotoxin contents in the resultant samples were less than 0.02 ng/mg protein (QCL-1000; Daiichi Pure Chemicals, Tokyo, Japan), respectively.

\section{Induction of Arthritis}

MSU crystals were prepared according to the methods reported by McCarty and Faires (1963). All animal experiments were performed under the guidelines and permission of the Animal Care Committee of Kumamoto University School of Medicine. Female New Zealand white rabbits (weight, 2.2 to $2.5 \mathrm{~kg}$ ) were anesthetized by pentobarbital sodium $(30 \mathrm{mg} / \mathrm{kg}$ ) given intravenously, and MSU crystals $(10 \mathrm{mg} / 500 \mu \mathrm{l}$ saline) were injected in the presence of $10 \mathrm{U}$ of polymixin B (PB; Pfizer Pharmaceutical, Tokyo, Japan) into the knee joints through the suprapatellar ligament. At various intervals after the injection, rabbit were anesthetized, bled, and killed. The knee joints were washed with $1 \mathrm{ml}$ saline and the joint fluids were collected and centrifuged at $6,000 \times g$ for 1 minute at $4^{\circ} \mathrm{C}$. The cell-free synovial fluids were harvested and stored at $-80^{\circ} \mathrm{C}$ until subsequent analyses. Cell pellets were resuspended in saline and the numbers were counted. Differential cell analysis was made after Wright-Giemsa staining.

\section{Blocking the Activities of Cytokines}

To neutralize $\mathrm{TNF} \alpha$, IL-1 $\beta$, IL-8, and $\mathrm{GRO} \alpha$ activity in knee joints, $10 \mu \mathrm{g}$ of anti-TNF $\alpha$ mAb, IL-1Ra, anti-IL-8 IgG, anti-GRO $\alpha$ mAb were injected simultaneously with MSU crystals in a total volume of $500 \mu$ l through the suprapatellar ligament into knee joints. Ten micrograms of each reagent were regarded as sufficient, because higher doses (30 to $100 \mu \mathrm{g}$ ) did not further reduce leukocyte infiltration (not shown). These agents were randomly chosen and injected into bilateral knee joints. Injection of inhibitors into one side of knee joints did not affect the leukocyte infiltration induced in the contralateral knee joints. Ten micrograms of mouse $\lg G$ and preimmune goat $\lg G$ were used as controls. The control IgG did not induce leukocyte infiltration and cytokine production. Leukocyte influx and cytokine/chemokine level between MSU crystals alone and MSU crystals plus control IgG were very similar (not shown). Therefore, injection of MSU crystals alone was used as control.

\section{Preparation of Neutrophil-Depleted Rabbits}

Neutrophil-depleted rabbits were prepared by intravenous injection of $0.75 \mathrm{mg} / \mathrm{kg}$ vinblastine (Sigma Chemical, St. Louis, Missouri) 3 days before the experiments (Rosenshein et al, 1979). The numbers of peripheral leukocytes before and after vinblastine treatment were $12.7 \pm 0.7 \times 10^{6} / \mathrm{ml}$ and $3.4 \pm 0.8 \times$ $10^{6} / \mathrm{ml}$, respectively. The majority of leukocytes in neutrophil-depleted rabbits were mononuclear cells, whereas $30 \%$ to $50 \%$ of the leukocytes in normal rabbits were neutrophils.

\section{Immunohistochemistry}

Freshly isolated synovial tissues were fixed in $4 \%$ paraformaldehyde, dehydrated, embedded in paraffin, and $4-\mu \mathrm{m}$-thick sections were made. After blocking 
endogenous peroxidase with $0.3 \% \mathrm{H}_{2} \mathrm{O}_{2}$ in methanol, the tissue sections were treated with $5 \%$ normal rabbit serum and incubated overnight at $4^{\circ} \mathrm{C}$ with goat polyclonal anti-rabbit GRO $\alpha$ IgG. Preimmune goat IgG was used as a control. After washing, the sections were then incubated for 30 minutes with $5 \mu \mathrm{g} / \mathrm{ml}$ biotinylated rabbit anti-goat IgG (Vector Laboratories, Burlingame, California), rinsed, and incubated for 30 minutes with avidin-biotin-peroxidase complex (Vector). As a chromogen, diaminobenzidine (DAB; Wako Chemical Industries, Osaka, Japan) was used. Counterstaining was performed with hematoxylin.

\section{Measurement of Cytokines}

The IL-1 $\beta$ and IL-8 levels were measured quantitatively by ELISA, as described (Matsukawa et al, 1997). The detection limits were $10 \mathrm{pg} / \mathrm{ml}$ and $30 \mathrm{pg} / \mathrm{ml}$, respectively. GRO $\alpha$ was measured quantitatively by time-resolved fluoroimmunoassay, as described previously (Matsukawa et al, 1999). The detection limit was $3 \mathrm{pg} / \mathrm{ml}$. TNF $\alpha$ activity was determined by L929 cell cytotoxic assay, as described (Flick and Gifford, 1984). This cytotoxic assay was specific to $T N F \alpha$, as preincubation of the samples with neutralizing antiTNF $\alpha$ mAb completely abolished the cytotoxicity for L929 cells. The detection limit was $30 \mathrm{pg} / \mathrm{ml}$. Synovial fluids were digested with $10 \mathrm{U} / \mathrm{ml}$ hyaluronidase (Sigma) for 1 hour at $37^{\circ} \mathrm{C}$ before assays.

\section{Statistics}

Statistical significance was evaluated by two-tailed unpaired Student's $t$ test; $p<0.05$ was regarded as statistically significant. All data were expressed as mean $\pm \mathrm{SE}$.

\section{Acknowledgements}

We thank Mr. S. Kudo, Ms. M. Kagayama, and Ms. T. Maeda for their technical assistance.

\section{References}

Alexander AG (1986). The pain of acute gout. Am J Med 80:133.

Baggiolini M, Dewad B, and Moser B (1997). Human chemokines: An update. Annu Rev Immunol 15:675-705.

Flick DA and Gifford GE (1984). Comparison of in vitro cell cytotoxic assays for tumor necrosis factor. J Immunol Methods $68: 167-175$.

Fujiwara K, Matsukawa A, Ohkawara S, Takagi K, and Yoshinaga M (2002). Functional distinction between CXC chemokines, interleukin-8 (IL-8), and growth related oncogene (GRO)alpha in neutrophil infiltration. Lab Invest 82:1523.

Fukumoto T, Matsukawa A, Yoshimura T, Edamitsu S, Ohkawara S, Takagi K, and Yoshinaga M (1998). IL-8 is an essential mediator of the increased delayed-phase vascular permeability in LPS-induced rabbit pleurisy. J Leukoc Biol 63:584-590.
Golds EE, Mason P, and Nyirkos P (1989). Inflammatory cytokines induce synthesis and secretion of gro protein and a neutrophil chemotactic factor but not beta 2-microglobulin in human synovial cells and fibroblasts. Biochem J 259:585588.

Gordon TP, Terkeltaub R, and Ginsberg MH (1988). Gout: Crystal-induced inflammaion. In: Gallin JI, Goldstein IM, and Snyderman R, editors. Inflammation: Basic principles and clinical correlates. New York: Raven Press, 775-783.

Hall DA, Beresford IJ, Browning C, and Giles H (1999). Signalling by CXC-chemokine receptors 1 and 2 expressed in $\mathrm{CHO}$ cells: A comparison of calcium mobilization, inhibition of adenylyl cyclase and stimulation of GTPgammaS binding induced by IL-8 and GROalpha. Br J Pharmacol 126:810-818.

Haranaka K, Satomi N, Sakurai A, and Nariuchi H (1985). Purification and partial amino acid sequence of rabbit tumor necrosis factor. Int J Cancer 36:395-400.

Kunkel SL, Lukacs N, and Strieter RM (1995). Chemokines and their role in human disease. Agents Actions Suppl 46:11-22.

Luster AD (1998). Chemokines-chemotactic cytokines that mediate inflammation. $\mathrm{N}$ Engl J Med 338:436-445.

Matsukawa A, Mori S, Ohkawara S, Maeda T, Tanase S, Edamitsu S, Yanagi F, and Yoshinaga M (1992). Production, purification and characterization of a rabbit recombinant IL-1 receptor antagonist. Biomed Res 13:269-277.

Matsukawa A, Yoshimura T, Fujiwara K, Maeda T, Ohkawara $S$, and Yoshinaga M (1999). Involvement of growth-related protein in lipopolysaccharide-induced rabbit arthritis: Cooperation between growth-related protein and IL-8, and interrelated regulation among TNFalpha, IL-1, IL-1 receptor antagonist, IL-8, and growth-related protein. Lab Invest 79: 591-600.

Matsukawa A, Yoshimura T, Maeda T, Takahashi T, Ohkawara S, and Yoshinaga M (1998). Analysis of the cytokine network among tumor necrosis factor alpha, interleukin1beta, interleukin-8, and interleukin-1 receptor antagonist in monosodium urate crystal-induced rabbit arthritis. Lab Invest 78:559-569.

Matsukawa A, Yoshimura T, Miyamoto K, Ohkawara S, and Yoshinaga M (1997). Analysis of the inflammatory cytokine network among TNF alpha, IL-1 beta, IL-1 receptor antagonist, and IL-8 in LPS-induced rabbit arthritis. Lab Invest 76:629-638.

McCarty DJ (1994). Crystals and arthritis. Dis Mon 40:253299.

McCarty DJ and Faires JS (1963). A comparison of the duration of local anti-inflammatory effects of several adenocorticosteroid esters: A bioassay technique. Curr Ther Res 5:284-290.

Miguelez R, Palacios I, Navarro F, Gutierrez S, SanchezPernaute O, Egido J, Gonzalez E, and Herrero-Beaumont G (1996). Anti-inflammatory effect of a PAF receptor antagonist and a new molecule with antiproteinase activity in an experimental model of acute urate crystal arthritis. J Lipid Mediat Cell Signal 13:35-49.

Mo JS, Matsukawa A, Ohkawara S, and Yoshinaga M (1999). Role and regulation of IL-8 and MCP-1 in LPS-induced uveitis in rabbits. Exp Eye Res 68:333-340. 
Mo JS, Matsukawa A, Ohkawara S, and Yoshinaga M (2000). CXC chemokine GRO is essential for neutrophil infiltration in LPS-induced uveitis in rabbits. Exp Eye Res 70:221-226.

Nishimura A, Akahoshi T, Takahashi M, Takagishi K, Itoman M, Kondo H, Takahashi Y, Yokoi K, Mukaida N, and Matsushima K (1997). Attenuation of monosodium urate crystalinduced arthritis in rabbits by a neutralizing antibody against interleukin-8. J Leukoc Biol 62:444-449.

Rae SA, Davidson EM, and Smith MJ (1982). Leukotriene B4, an inflammatory mediator in gout. Lancet 2:1122-1124.

Rollins BJ (1997). Chemokines. Blood 90:909-928.

Rosenshein MS, Price TH, and Dale DC (1979). Neutropenia, inflammation, and the kinetics of transfused neutrophils in rabbits. J Clin Invest 64:580-585.

Rosenthale ME, Dervinis A, Kassarich J, and Singer S (1972). Prostaglandins and anti-inflammatory drugs in the dog knee joint. J Pharm Pharmacol 24:185-198.

Terkeltaub R (1993). Pathogenesis and treatment of crystalinduced inflammation. In: McCarty D and Koopman W, editors. Arthritis and allied conditions. Philadelphia: Lea \& Febiger, 1819-1833.
Terkeltaub R, Baird S, Sears P, Santiago R, and Boisvert W (1998). The murine homolog of the interleukin-8 receptor CXCR-2 is essential for the occurrence of neutrophilic inflammation in the air pouch model of acute urate crystal-induced gouty synovitis. Arthritis Rheum 41:900-909.

Terkeltaub R, Zachariae C, Santoro D, Martin J, Peveri P, and Matsushima K (1991). Monocyte-derived neutrophil chemotactic factor/interleukin-8 is a potential mediator of crystalinduced inflammation. Arthritis Rheum 34:894-903.

Webster ME, Maling HM, Zweig MH, Williams MA, and Anderson WJ (1972). Urate crystal induced inflammation in the rat: Evidence for the combined actions of kinins, histamine and components of complement. Immunol Commun 1:185-198.

Weinberger A (1995). Gout, uric acid metabolism, and crystal-induced inflammation. Curr Opin Rheumatol 7:359363.

Zlotnik A and Yoshie O (2000). Chemokines: A new classification system and their role in immunity. Immunity 12:121127. 